\title{
An Improved Function Optimization Problem (IFOP) of Evolutionary Programming Algorithm - A Survival Paper
}

\author{
R. Karthick \\ Assistant Professor, \\ Karpagam Institute of Technology
}

\author{
S. Saravanan \\ Assistant Professor, \\ Karpagam Institute of Technology
}

\begin{abstract}
Evolutionary Algorithms are based on some influential principles like Survival of the Fittest and with some natural phenomena in Genetic Inheritance. The key for searching the solution in improved function optimization problems are based only on Selection and Mutation operators. This paper reflects on Survival selection schemes specifically like Truncate Selection, Proportionate Selection, Tournament Selection and Ranking Based Selection. In this paper we calculate the best fittest value among the populations which is generated.
\end{abstract}

\section{Keywords}

Evolutionary Programming, Function Optimization.

\section{INTRODUCTION}

The principles which is inspired by Darwinian is based on the evolution and natural selection, a significant amount of computational models and methods have been studied under the umbrella of evolutionary computation[1] The evolutionary algorithm is a population based algorithm. To solve the function optimization or parameter optimization problems the Evolutionary strategies are used. The evolutionary algorithm uses the selections schemes to focus the selection of higher or best fitness from the population. For standard selection schemes this is controlled by appropriately setting the parameters that govern the selection pressure on the individuals [2]. The major steps followed in calculating the fitness are i) Mutation operation is performed in current population ii) Identification of Individual selection for the next generation. The survival of the fittest among the natural populations is based on natural selection strategies. By mimicking this process, evolutionary algorithms are able to 'evolve' solutions to real world problems, if they have been suitable encoded The Evolutionary algorithms have been applied to solve some practical problems like global optimization, machine learning. The offspring are produced based on the operations performed between two randomly generated populations. For calculating and choosing the best solutions among the populations, various selections like Tournament and Rank Based Selection Strategies are used. Individuals live one period (one generation), compete between themselves, and the better ones (Fitter for environments) are reproduced. Offspring's differ from the parents individuals because the genetic operators act during reproduction [4]. This paper mainly focused on calculating the best solution among various two randomly generated populations.

\section{EVOLUTIONARY PROGRAMMING STRATEGIES}

Evolutionary Strategies were developed as a method to solve parameter optimization problems [5]. To seek the evolution of artificial intelligence, the evolutionary programming had developed for the ability to predict the changes in the environment. The individuals undergoes a Gaussian distribution method of addition zero mean Gaussian variable of standard deviation. The parent solution for the next generation can be obtained from the Survival of the fittest from the parent and the offspring [6]. The next generations are called as offspring. The procreation of a population in the general computer programs are based Evolutionary programming techniques. GP has been mainly intended for discovering computer programs that are able to solve particular classes of optimization problems [7]. The nearer optimum solution can be obtained by use of evolutionary search algorithm iteratively. [8]. In the Genetic algorithm the set of data's (represented in numbers) called as "Chromosomes" and the elements in the data are called as "genes" The survival of the Fittest is identified by calculating the function.

\subsection{Genetic Algorithm and its Operators}

The operation of GA's begins with a population of a random string representing design or decision variables. To create a new population or offspring of the points here we need to follow the three methods such as reproduction, crossover and mutation [9]. To start with the function optimizations in the genetic algorithm we need to set the population in the encoded format and define it as members. Cross over and mutation, operators based on reproduction, are used to create the next generation.[10] For creating next generation, crossover plays a vital role here by combining the members in the current generated population. The mutation systematically changes elements of a solution from the current generation in order to create a member of the next generation [10]. To find the best solution or survival of the fittest, genetic algorithms uses the cross over and mutation and performs the operation. Genetic algorithms are well suited for searching complex, highly nonlinear spaces because they avoid becoming trapped in a local minimum [11].

\subsection{Proposed Selection with Problem Description}

The problem is that identifying the survival of the fittest (Best solution), from the randomly generated populations. After identifying the best solution the tournament selection is applied to the new generations (offspring's) and the survival 
of the fittest is identified. The minimum best population is identified and grouped in an order, and at one stage there will be no changes in the offspring which is generated after the tournament selection. Various rules have been described to identify the offspring for the next generation.

- Rule 1: The newly generated individuals are given ranked according to the tournament operation which is applied.

- Rule 2: All the solutions are positioned at one location and one best solution is taken among them

- Rule 3: With a certain probability, collective solutions are taken for creating a subsequent selection.

\section{BASIC OPERATIONS ON EP}

This paper mainly focuses on IFOP (Improved Function Optimization Problem). The IFOP includes three main elements $\mathrm{A}, \mathrm{B}$ and $\mathrm{F}$ where $\mathrm{A}$ is considered as one population and $\mathrm{B}$ is considered to be another population and $\mathrm{F}$ is considered as function such as $\mathbf{F}->\mathbf{A}+\mathbf{2} * \mathbf{B}$. A population is considered to be $\mathrm{n}$ positions were $\mathrm{n}$ is the population size. Each population is generated based on the size which is defined such as

\section{$A=1 *$ rand $(1,10)$ and $B=2 *$ rand $(1,10)$}

After the population is generated the evaluation is done between the population and best solution is obtained. The following formula is used to generate the fittest

\section{$\mathbf{F}=\mathbf{A}+2 * \mathbf{B}$}

The basic steps for implementing the IFOP are depicted below. When populations are generated, the F (formula) is applied between the two populations and identifies the highest win which is used to form offspring and from the offspring the tournament selection operation is applied and best fittest is chosen

\subsection{Process Involved}

S1. Generate two populations randomly between some ranges S2. From for example $1 *$ rand $(1,10)$ and $2 *$ rand $(1,10)$ the population generation calculate the fitness function based on our formulae which is given

S3. Sort the population in decreasing order based on the fitness.

S4. Perform the operation till all the elements get sorted. S5. Perform a pair wise tournament between the fitness values and replace the new fitness value with the old fitness values. S6. Plot the graph based on the result which is generated.

\section{ALGORITHM}

//Input: A and B are generated randomly

//Output: Best solution

Begin

$\mathrm{A} \leftarrow 1$ *rand $(1,10)$

$\mathrm{B} \leftarrow 2 *$ rand $(1,10)$;

For generating the solution for every element in the population

$$
\begin{aligned}
& \text { For } \mathrm{i}=1: 10 \\
& \text { End for } \\
& \text { Return min }(\mathrm{F})
\end{aligned}
$$

End

\subsection{Genetic Algorithm approach to IFOP}

Genetic Algorithm approach is engaged to optimally select the minimum best solution from the randomly generated population. To identify the global minimum search space the traditional genetic algorithms were used to explore the solutions. There are various ways in genetic algorithm to improve the performance of evolutionary programming. Here we follow Cross over and Mutation. The cross over encapsulates various elements in the current generation and creates an offspring (New Member). The mutation is nothing but sudden change in the element of the current population which is generated. Genetic algorithm are also well suited for searching complex, highly non-linear spaces because they avoid becoming trapped in a local minimum [11] In the evolutionary computation method, the fitness estimation is very much important, were the individual are considered as values and encodes only part of the solution. There are some basic outlines of genetic algorithm which are as follows

- Initialize the population

- Evaluating the fitness

- Creating a new population

- Selection

- Crossover

- Mutation

- Placing the new offspring which is generated

- Replacement by selection operations

- Testing

- Iterations for all populations.

The GA's are known as Search algorithms, which are based on the phenomenon of natural selection and natural genetics. From our problem, a certain population is chosen (coding format) and the candidate for the solution to the optimization problem. The first form is nothing but we need to generate the population randomly. From the randomly generated populations we need to generate the offspring. The offspring inherits the characteristics of parents from which they have generated. After all the offspring's are generated a tournament operations is performed between them so that the low fitness is replaced by the best fitness.

\subsection{Tournament Selection}

The process involved in the tournament selection is as follows,

1. Two solutions are taken randomly from the available population

2. Fitness is compared between the chosen populations

3. Best fitness replaces the least fitness

4. This determines the tournament selection selects the best individual in each tournament

For example consider the below table,

Table1: Population and Fitness.

\begin{tabular}{|c|c|c|}
\hline $\mathbf{A}$ & $\mathbf{B}$ & Fitness \\
\hline 2.3845 & 1.5461 & 5.4766 \\
\hline 2.8075 & 1.047 & 4.9646 \\
\hline 1.5678 & 4.5756 & 10.719 \\
\hline 2.6404 & 1.7966 & 6.2335 \\
\hline 0.5189 & 2.1949 & 4.9087 \\
\hline 2.9392 & 3.6458 & 10.2308 \\
\hline
\end{tabular}




\begin{tabular}{|c|c|c|}
\hline 0.8143 & 2.1376 & 5.0896 \\
\hline 0.757 & 2.8769 & 6.5108 \\
\hline 2.6272 & 1.2591 & 5.1455 \\
\hline 2.2119 & 4.9533 & 12.1186 \\
\hline
\end{tabular}

From the above population chose two solutions randomly e.g. 10.719 and 12.1186. Here the best solution is 12.1186 it replaces the 10.2308 like this the iteration happens for all the individuals and best solution is found.

\subsection{Crossover and Mutation}

Here, we use 1 point crossover. The crossover is used to create a new offspring. From the two populations the two offspring is generated.

The below table 2 depicts this,

Table2: Single point Crossover

\begin{tabular}{|l|l|}
\hline Population1 & 11011001110001 \\
\hline Population2 & $11011 \quad 110000011$ \\
\hline Offspring1 & $11011 \quad 110001111$ \\
\hline Offspring2 & 11011000110011 \\
\hline
\end{tabular}

After the operations which are performed in the crossover, the next step is we need to go for mutation. The mutation is nothing but sudden changes in the offspring. That is, in the binary encoding, the mutation occurs is the values of 0 changes to 1 and 1 changes to 0 .

Table3: Mutation.

\begin{tabular}{|l|l|}
\hline Original Offspring1 & 1100011001101 \\
\hline Original Offspring2 & 1101111001100 \\
\hline Mutated Offspring1 & 110111001101 \\
\hline Mutated Offspring2 & 1111011001100 \\
\hline
\end{tabular}

The genetic algorithm cycle is depicted in the below figure.

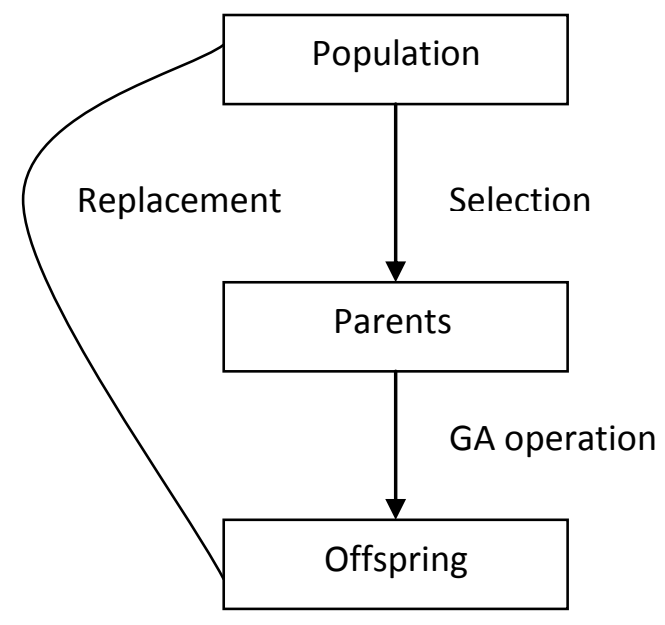

Fig1: GA Cycle

\section{RESULTS AND DISCUSSIONS}

The result which is represented in below figure depicts the identification of best solution. The graph is plotted by collecting all the best solutions which is obtained after performing all the operations. For the populations $\mathrm{A}$ and $\mathrm{B}$ which is given eg. 10 populations are generated and fitness is calculated between them. By applying the tournament operation the lower fitness values is replaced by higher fitness values.
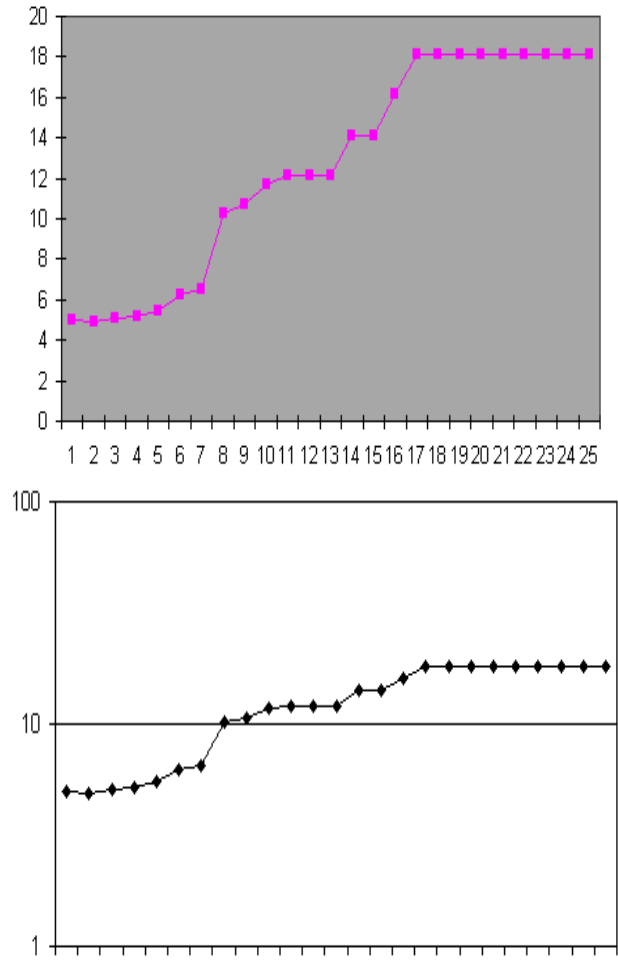

12345678910111213141516171819202122232425

Fig2: Graphical Representation of Best solutions

\section{CONCLUSION}

This paper is concerned with the improved function optimization techniques (IFOP), which deals with identification of the best solution. The algorithms which is presented in GA and steps involved in EP are very much help to solve the problem of function optimization. The final thing by the real time example for these optimization techniques from the plotted graph is that from Monkey to Human. After Human no more generation is there, so after replacing of all the best solution no more work is need for any replacements. The most convenient of this problem is we can extend the parameters which have been declared and the function can also be added with additional parameters. Finally we can conclude that, identifying the best solutions based on the survival of the fittest from various populations.

\section{REFERENCES}

[1] X.Yao and Y.Xu, "Recent Advances in Evolutionary Computations" Int. J. Compt. Sci and technology

[2] "Tournament versus Fitness Uniform Selection" by Shane Legg, Marcus Hutter, Akshat Kumar.

[3] "Natural Computation for Business Intelligence from Web Usage Mining".by Ajith Abraham 
[4] K-Model "An Evolutionary Algorithm with New Schema of Representation" by Halina Kwasnika.

[5] Schwefel H.P "On the Evolution of Evolutionary Computation".

[6] "Evolutionary Computation" Marc Schoenauer and Zbigniew Michalewicz.

[7] "Evolving Evolutionary Algorithms for function Optimization" by Mihai Oltean

[8] "Evolutionary Algorithms for optimizing bridge deck rehabilitation"'

[9] "Genetic Algorithm" by Tom V.Mathew

[10] Coercion through optimization: A Classification of Optimization Techniques by sarah wazirud, David C. Brogan and Paul F. Reynolds Jr.

[11] Y.Carson and A.Maria: "Simulation Optimization: Methods and Applications proceedings of the 1977 Winter Simulation Conference, 1977

[12] “ Developmental Evaluation in Genetic Programming: A Position Paper" Tuan Hao Hoang, Student Member, IEEE, RI (Bob) McKay, Senior Member, IEEE, Daryl Essam, and Xuan Hoai Nguyen
[13] Evolutionary Algorithm by “ Zbigniew Michalewiz, Robert Hinterding, Maciej Michalewicz

[14] "Genetic Solution for Building Design", by S.Balasubramaniam, C.Kalairaja, S.Karthikeyan, N.Venkateswaran.

[15] "Selective Mutation for Genetic Algorithm", Sung Hoon Jung in World Academy of Science, Engineering and Technology 562009.

[16] "Predictive Analytics using Genetic Algorithm for Efficient Supply chain Inventory Optimization", by P.Radhakrishnan, Dr.V.M. Prasad, N. Jeyanthi.

[17] "Adaptive Particle Swarm Optimization (APSO) for multimodal function optimization", Md. Sakhawat Hossen, Fazle Rabbi, Md. Mainur Rahman.

[18] "Different Aspects of Evolutionary Algorithms, Multi Objective Optimization Algorithms and Application Domain”, Dhirendra Pal Singh, Ashish Khare.

[19] "Genetic Algorithm- an Approach to solve Global Optimation”, Prathibha Bajpai and Dr. Manoj Kumar.

[20] "Penalty Function Methods for Constrained Optimization with Genetic Algorithms", Ozgur Yeniay. Mathematical and Computational Applications, Vol. 10, No. 1, PP 4556,2005 